\title{
An Efficient Image Distortion Correction Method for an X-ray Digital Tomosynthesis System
}

\author{
J.Y. Kim \\ Dept. of Mechatronics Engineering, \\ Tongmyong University of Information Technology, \\ 535 Yongdang-dong, Nam-gu, Busan 608-711, Korea \\ kjy97@tit.ac.kr
}

\begin{abstract}
Among X-ray cross-sectional imaging methods, digital tomosynthesis (DT) is very useful to PCB inspection because it can obtain a cross-sectional image of a local inspection area quickly. The image intensifier, which is usually used in DT systems, distorts X-ray images in shape and intensity. Therefore, image distortion correction is one of the most important issues in realizing a DT system. This paper presents an image distortion correction method to acquire an arbitrary cross-sectional image of an object by using a distance ratio function in an X-ray DT system. The method uses a simplified distortion model made by a distance ratio function in intensity correction, and by the $2 \mathrm{D}$ point mapping polynomials in shape correction.
\end{abstract}

\section{Introduction}

X-ray technology has been widely used in many industrial applications for inspecting inner defects which can hardly be found by normal vision systems. PCB solder joint inspection such as ball grid array (BGA) or flip chip array (FCA) is one of the applications that require such an X-ray inspection system[1,2]. An X-ray crosssectional image can be obtained from two or more images projected from different directions by the methods such as tomography, laminography, or digital tomosynthesis.

Tomography has been mainly used in medical area, but recently it is being applied in industrial fields such as precision inspection of casting products[3]. Laminography was originated by Bocage[4]. Its principle comes from the geometric focusing effect by a synchronized motion between an X-ray source and a detector, which is shown in Fig. 1. Digital tomosynthesis is a digital version of laminography, where a set of images of different views are stored and synthesized through computational operations in a computer[5]. It is one of the most useful X-ray cross-sectional imaging methods for PCB inspection because it can obtain a cross-section of a local inspection area quickly. Thus it has been often applied to PCB solder joint inspection[5-8]. However, the shape and the intensity of the X-ray images obtained by DT are distorted because of the image intensifier used in DT systems. This distortion breaks the correspondences between those images and prevents us from acquiring accurate cross-sectional images. Therefore, image distortion correction is one of the most important issues in realizing a DT system. 


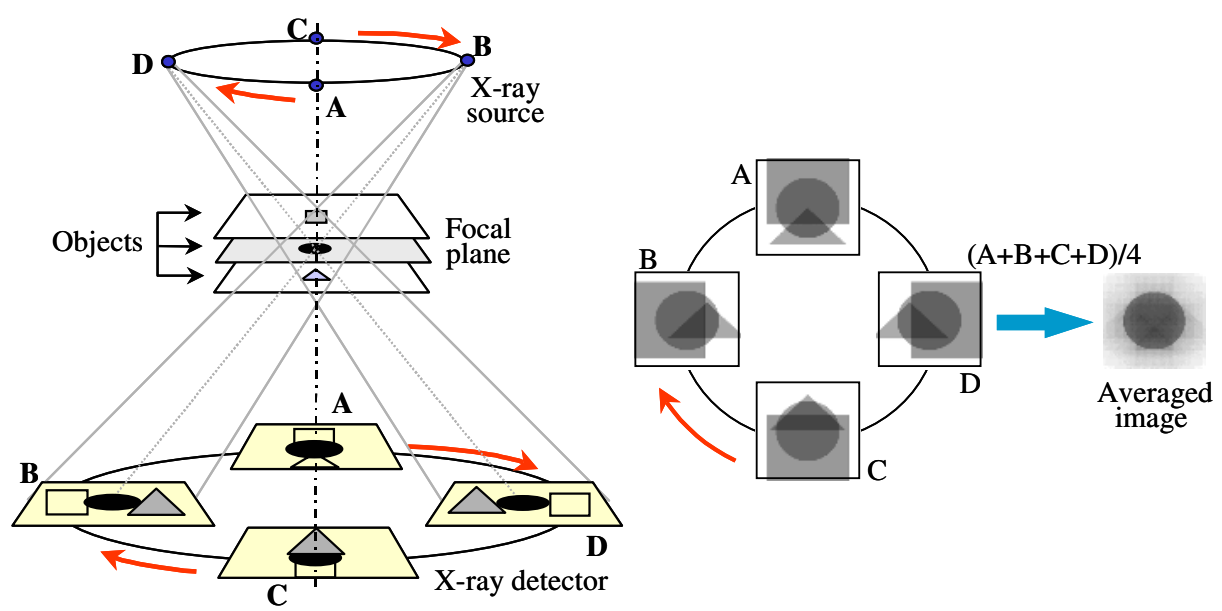

Fig. 1. The principle of Laminography and Digital Tomosynthesis

In this paper, an image distortion correction method for an X-ray DT system is presented. The method uses a simplified distortion model that is built by uniformly spaced grids and their distorted images. The intensity distortion model is based on the distance ratio function between two grids, and the shape distortion model is based on the two-dimensional point mapping polynomials. Also this paper performs a series of experiments to acquire PCB solder joint images by the presented correction method.

\section{System Configuration and Image Distortion}

Fig. 2(a) shows a configuration of the developed X-ray DT system, which is composed of a scanning X-ray tube, an image intensifier, a view selector and a zoom camera. An image intensifier with a large input screen is used as an X-ray detector so as to get all images projected at various directions. The area of interest of a PCB is projected on a circular trajectory on the image intensifier as the X-ray is steered on the trajectory, and eight or more images are sequentially acquired by the zoom camera through a view selector. A galvanometer or a rotating prism can be used as a view selector. The captured images are saved in the digital memory of a computer, and then synthesized to generate a cross-sectional image.

The curved image input surface of the intensifier, however, distorts both of the shape and the intensity of the X-ray images. Fig. 2(b) shows eight distorted images of an uniformly spaced grid pattern projected onto the image intensifier according to the steered X-ray source location. It is shown that the images are distorted more severely in the peripheral area than the central area. It is not possible to get an accurate crosssectional image from these distorted images, since the correspondences between the images are not maintained anymore. 


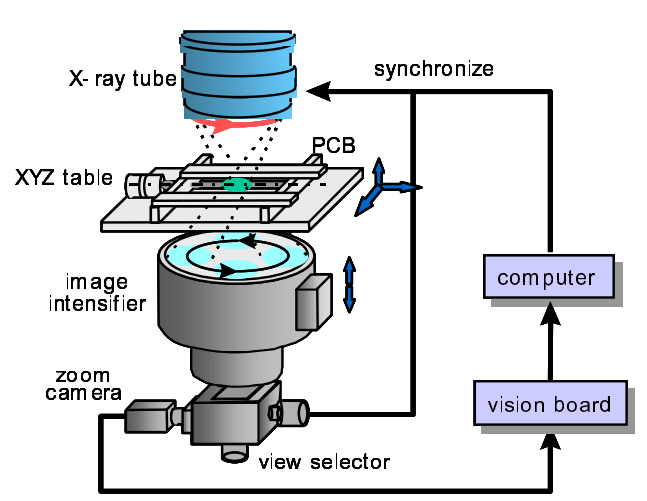

(a) a schematic

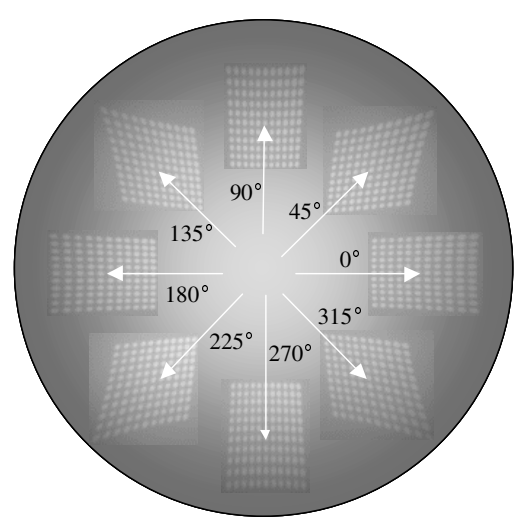

(b) distorted images from the system

Fig. 2. The configuration of an X-ray Digital Tomosynthesis system

\section{Intensity Distortion Correction by Using a Distance Ratio Function}

The central area of the image intensifier is brighter than the peripheral area, since the incident angle of the X-ray to the curved input surface of the intensifier varies with the incident locations. In each projected image, therefore, intensity is the highest in the vicinity of the image intensifier center and gets lower toward the peripheral area. To compensate for the distorted intensity and make it uniform over the image, the distorted intensity should be scaled on the same level with an intensity value, for example, the maximum intensity level of the image. In order to do it, intensities are sampled over the image area and the distribution is modeled numerically.

The distorted intensity $\Phi_{d}(i, j)$ at a point $(i, j)$ of an image can be corrected to the compensated intensity $\Phi_{c}(i, j)$ by dividing by the distance ratio function $f\left(L_{d}\right)$ for the point, as given in Eq. (1).

$$
\begin{gathered}
\Phi_{c}(i, j)=\Phi_{d}(i, j) / f\left(L_{d}\right) . \\
f\left(L_{d}\right)=c_{0}+c_{1} L_{d}+c_{2} L_{d}^{2}+c_{3} L_{d}^{3} . \\
L_{d}(i, j)=\sqrt{\left(i-i_{H}\right)^{2}+\left(j-j_{H}\right)^{2}}
\end{gathered}
$$

where $L_{d}(i, j)$ is defined as the distance from the highlight point $H=\left(i_{H}, j_{H}\right)$ to the point $(i, j)$. The distance ratio function $f\left(L_{d}\right)$ has a value decreasing with $L_{d}$ between 0 and 1 , thus plays a role of correcting an intensity $\Phi_{d}$ to $\Phi_{c}$. To build the ratio function $f\left(L_{d}\right), 10$ intensity values are sampled from the 10 small areas on the path from the point $\mathrm{H}$ to the point $\mathrm{L}$, as shown in the Fig. 3. The coefficients of the polynomial $f\left(L_{d}\right)$ can be determined by least square method. 

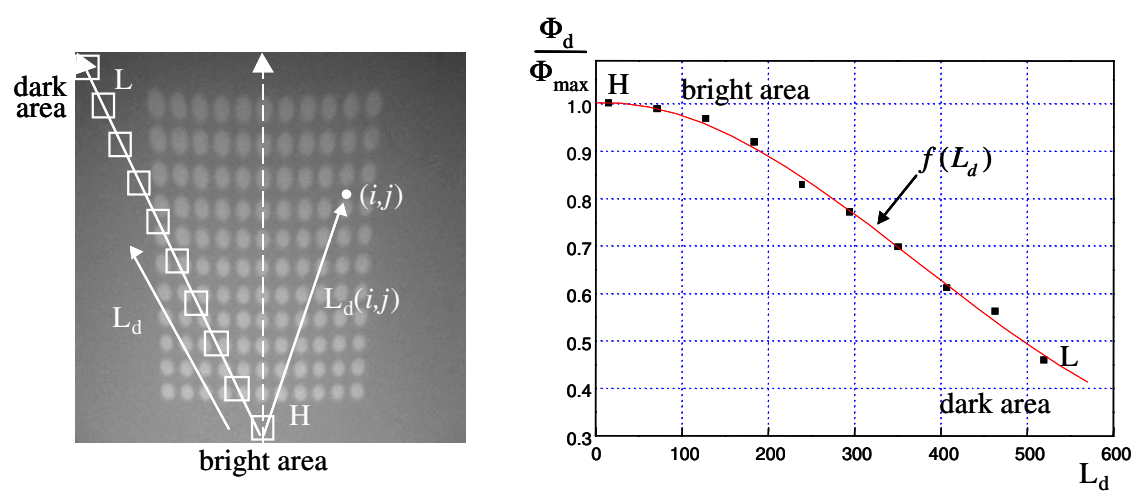

Fig. 3. Distorted intensity profile for a distance ratio function

\section{Shape Distortion Correction by Using a Simplified Polynomial Model}

The shape distortion can be corrected by finding a mapping relationship between the uniformly spacing grid image and its distorted image. Thus, a point $(i, j)$ in the original undistorted image is mapped to a point $(x, y)$ in the distorted image by the mapping relation, as shown in Fig. 4. As the sample data for distortion modeling, this paper used the data sets which consist of the uniformly spaced grid points of $11 * 11$. There are two features in the shape distortion. One is that the distorted images are always symmetric with the projection center-line of the x-ray. Another one is that the peripheral area of the image intensifier is more elongated than the central area. Based on the two features, the mapping relationship can be modeled and represented by the following equations.

$$
\begin{gathered}
y_{k}(i, j)=\alpha_{k}(j)\left\{x(i, j)-x_{c}\right\}^{2}+P_{k}(j) . \\
x_{k}(i, j)=\beta_{k}(j) \cdot i+i_{c} \cdot \\
P_{k}(j)=p_{0}^{k}+p_{1}^{k} \cdot j+p_{2}^{k} \cdot j^{2}+p_{3}^{k} \cdot j^{3} \\
\alpha_{k}(j)=a_{0}^{k}+a_{1}^{k} \cdot j+a_{2}^{k} \cdot j^{2}+a_{3}^{k} \cdot j^{3} . \\
\beta_{k}(j)=b_{0}^{k}+b_{1}^{k} \cdot j+b_{2}^{k} \cdot j^{2}+b_{3}^{k} \cdot j^{3}
\end{gathered}
$$

A distorted line, which is a distortion of an original horizontal line, is modeled by using a 2nd order polynomial as shown in Eq. (4). On the other hand, the $x$ coordinate values $x_{k}(i, j)$ of the distorted line are modeled as shown in Eq. (5). The parameters $P_{k}(j), \alpha_{k}(j), \beta_{k}(j)$ are functions of $j$, and they are modeled by using 3rd order polynomials as shown in Eq. (6). The coefficients $p_{0}^{k} \sim p_{3}^{k}, a_{0}^{k} \sim a_{3}^{k}, b_{0}^{k} \sim b_{3}^{k}$ are determined by using least square fitting. 


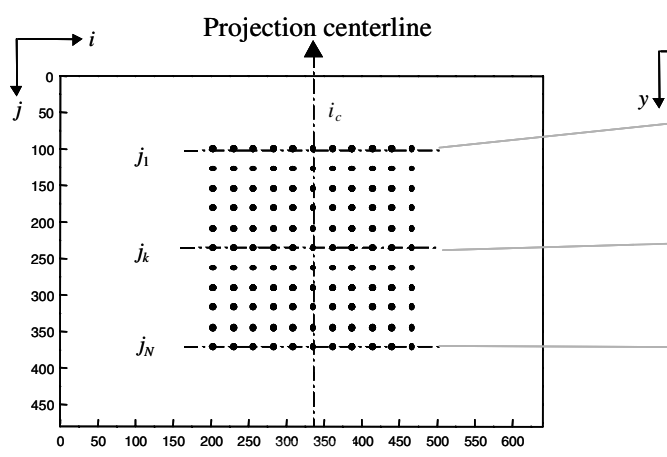

(a) original grid points

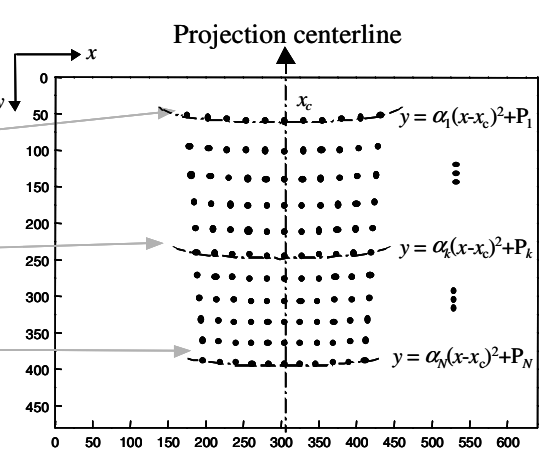

(b) distorted grid points

Fig. 4. Two-dimensional point mapping for shape distortion correction

\section{Experiments for PCB Solder Joint Image Acquisition}

A series of experiments to get the DT images of BGA was performed by using the Xray DT system shown in Fig. 2(a). In the acquired images shown in Fig. 5, the dark regions represent the cross-sections of the corresponding focal planes. The crosssection at the middle of the lead ball has the maximum diameter. These DT images were acquired by integrating the 8 images taken from 8 different off-axis images. In each image of them, the intensity and shape distortion was corrected by using the correction method presented in above sections. These cross-sectional images provide a good cue or a unique solution to inspect the inner defects of the objects such as BGA or J-lead typed chips.

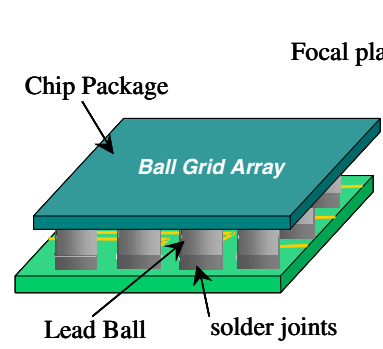

(a) BGA
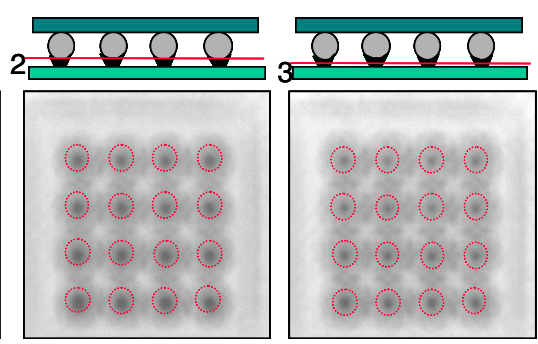

(b) DT images for the focal plane 1-3

Fig. 5. X-ray DT images of BGA

\section{Conclusions}

In order to obtain an arbitrary cross-sectional image with high quality in an X-ray DT system, this paper presented a method to correct the distortion of image intensity and image shape. The method uses a simplified distortion model made by a distance ratio 
function in intensity correction, and by the $2 \mathrm{D}$ point mapping polynomials in shape correction. And a series of experiments to get DT images of BGA was performed by using the presented correction method. The experimental results show that the presented correction method is effective in acquiring an arbitrary cross-sectional image of an object.

\section{References}

1. Adams, J.: X-ray laminography analysis of ultra fine pitch solder connections on ultra-thin boards. SPIE Integrated Circuit Metrology, Inspection, and Process Control V, Vol. 1464. (1991) 484-497

2. Moore, T. D., Vanderstraeten, D., Forssell, P.M., Three-dimensional x-ray laminography as a tool for detection and characterization of BGA package defects. IEEE Tr. on Components and Packaging Technologies, Vol. 25. No. 2. (2002) 224-229

3. Bossi, R. H. and Georgeson, G. E.: Casting development savings with X-ray computed tomography. Casting (1993) 181-188

4. Bocage, E. M.: French Patent 536464 (1922)

5. Rooks, M. and Sack, T.: X-ray inspection of flip chip attach using digital tomosynthesis. Circuit World, Vol. 21. No. 3. (1995) 51-55

6. Bord, S., Clement, A., Lecomte, J. C., Marmeggi, J. C.: An X-ray tomography facility for IC industry at STMicroelectronics Grenoble. Microelectronic engineering, Vol. 62. (2002) 1069-1075

7. Sumimoto, T., Maruyamay, T., Azuma, Y., Goto, S., Mondo, M., Furukawa, N., Okada, S.: Detection of defects at BGA solder joints by using X-ray imaging. 2002 IEEE Int. Conf. on Industrial Technology, Vol. 1. (2002) 238-241

8. Roh, Y. J., Ko, K. W., Cho, H. S., Kim, J. Y., Byun, J. E.: The calibration of X-ray digital tomosynthesis system including the compensation of the image distortion. SPIE Symp. on Intelligent Systems and Advanced Manufacturing VII, Vol. 3528. (1998) 248-259 